Check for updates

Cite this: RSC Adv., 2018, 8, 41422

Received 26th September 2018 Accepted 29th November 2018

DOI: 10.1039/c8ra07982j

rsc.li/rsc-advances

\title{
Effects of methyl jasmonate and melatonin treatments on the sensory quality and bioactive compounds of harvested broccoli
}

\author{
Feng Luo, Jia-Hui Cai, Xuan Zhang, Dong-Bing Tao, Xin Zhou, Qian Zhou, \\ Ying-Bo Zhao, Bao-Dong Wei, Shun-Chang Cheng and Shu-Juan Ji (D)*
}

\begin{abstract}
Harvested broccoli is prone to decline in quality with regard to its appearance and nutrition. In this study, freshly harvested broccoli was treated with methyl jasmonate (MeJA) and melatonin (MT) and stored at $20{ }^{\circ} \mathrm{C}$ and the changes in sensory qualities and bioactive compounds were analyzed. The control samples began yellowing on day 2, whereas MeJA and MT treatments delayed the yellowing by 2 and 4 days, respectively. Upon yellowing, sweetness and bitterness of control samples increased sharply, accompanied by the accumulation of bioactive compounds, except for sulforaphane; however, no significant change in volatile components was detected. When the samples started losing their green color, MeJA alleviated the bitterness while increasing the sweetness and sulforaphane content. The bitterness, astringency, umami level, and the content of sulfurous volatiles improved significantly in the MT-treated samples. Moreover, these samples showed high antioxidant activity; the protective effect on $\mathrm{V}_{\mathrm{C}}$ and carotenoids was extremely significant.
\end{abstract}

\section{Introduction}

Vegetables are important components of diet, supplying a multitude of nutrients required for health. Broccoli (Brassica oleracea $\mathrm{L}$. var. italica) has been the focus of much research ${ }^{1}$ because of its potential to provide health-promoting phytochemicals in the diet. Broccoli heads are fresh and green when they are harvested, but are prone to deterioration, which results in the yellowing of the florets and a rapid decrease in their nutritional content upon storage. Moreover, on deterioration, the flavor and aroma of broccoli can become unacceptable to customers. To improve the post-harvest quality of broccoli, treatment with a variety of exogenous compounds, such as 1methylcyclopropene, sugars, ethanol, salicylic acid, and $\mathrm{N}$ benzylaminopurine, has been investigated., ${ }^{2,3}$

Methyl jasmonate (MeJA) is a plant hormone and functions as a signaling molecule. ${ }^{4}$ It is also an important fragrance component of jasmine flowers. Exogenous application of MeJA can promote effective accumulation of nutrients in fruits and vegetables by induction of hormone signal transduction in cells. ${ }^{5}$ Kang et al. ${ }^{6}$ reported that MeJA treatment could significantly increase glucosinolate biosynthesis in broccoli florets. Recently, researchers found that $N$-acetyl-5-methoxytryptamine (melatonin, MT) was effective in removing free radicals from cells. ${ }^{7}$ Several metabolites formed by the interaction of MT with

Department of Food Science, Shenyang Agricultural University, No. 120 Dongling Road, Shenyang 110866, PR China. E-mail: jsjsyau@yeah.net; jsjsyau@sina.com; 403880675@qq.com; Fax: +86 (0) 24 88498337; Tel: +86 (o) 2488498337 free radicals were also found to be highly effective scavengers of the free radicals. ${ }^{8}$ Besides, MT also stimulated the activity of protective antioxidant enzymes (glutathione reductase, glutathione peroxidase, and superoxide dismutase). Under extreme stress conditions, MT could effectively restore the germination of cucumber seeds that has been exposed to chilling injury. ${ }^{9}$ Moreover, melatonin treatment effectively increased the tolerance of cabbage seeds to toxic copper ion stress, increased the herbicidal ability of rice and alleviated the germination of Pennisetum alopecuroides L. Spreng seeds under $\mathrm{NaCl}$ stress., ${ }^{\mathbf{9} 10}$ However, the use of MT as an effective treatment against oxidation has rarely been reported in studies on the postharvest quality of broccoli.

Sensory quality, which includes color, taste, aroma, and tissue morphology, is the most direct index used to measure the quality of fruits and vegetables. Aroma is determined by a complex mixture of volatile components emitted by a product, which are perceived by the taste receptor; it might be decided by the off-odor produced by deterioration of the product quality. Usually, aroma is analyzed by gas chromatography-mass spectrometry (GC-MS). The taste, which could be sour, salty, sweet, bitter, astringent, or umami, is perceived by the tongue in mammals. Electronic tongues, developed recently, can simulate the taste receptors of mammals, and can reflect the taste of products and help in determining the effects of different treatments on the taste of products. ${ }^{11}$

Broccoli heads are made up of dense buds. The newly harvested broccoli is green, with the fragrance of fresh vegetables and the unique flavor of cruciferous vegetables. At room 
temperature, the color of broccoli gradually turns yellow during the shelf life and its aroma and taste also change significantly. In severe cases, it produces off-odor, which seriously affects its commodity value. There have only been few reports on the flavor and volatile components of broccoli. Lv et al. ${ }^{12}$ surmised that Se treatment can have a positive effect in maintaining the quality of broccoli and in enhancing its sensory quality through the release of volatile compounds.

The beneficial roles of broccoli in the prevention of cancer, cardiovascular disease, tumor, and senescence are wellrecognized, and are associated with the health-promoting effects of the bioactive compounds present in it. ${ }^{13}$ The main bioactive compounds of broccoli are flavonoids, vitamin $\mathrm{C}$, carotenoids, and sulforaphane. ${ }^{14}$ The nutrient content and antioxidant activity were extremely important during postharvest broccoli, which was susceptible to yellowing after harvest, and has a negative impact on its nutritional and commercial value. ${ }^{15} \mathrm{UV}$, steam and ethanol treatments effectively inhibited the reduction of antioxidant activity in postharvest broccoli. ${ }^{\mathbf{1 6 - 1 8}}$ Glucosinolates are unique secondary metabolites of cruciferous plants, which can produce a series of important biologically active substances upon hydrolysis by myrosinase, of which, sulforaphane is an effective anticancer compound. ${ }^{19}$ Among the vegetables of the Brassica family, broccoli is the ideal material for obtaining sulforaphane and, therefore, its consumption is increasing.

The causes and mechanisms of yellowing of broccoli have been investigated in a number of studies; however, research on the changes in sensory quality and bioactive compounds of broccoli that accompany the yellowing process remains scant. In the present study, we determined the changes in the sensory quality and bioactive compounds accompanying the yellowing of untreated broccoli during the shelf life. We also investigated the effects of MeJA and MT treatment on the above-mentioned parameters of broccoli during its shelf life after harvest.

\section{Materials and methods}

\subsection{Plant material and treatments}

'Naihan-Youxiu' broccoli heads were harvested at a commercial field in Shenyang, Liaoning Province, China. The standard for broccoli harvest was tight florets with buds of uniform size and color. Two or three leaves were left intact at the base of the ballflower. The harvested products were placed in plastic boxes, with head pointing upwards and no stacking, and were immediately transported to the laboratory where they were selected based on similar maturity and size and absence of physical damage and pests. The samples were divided into three groups (control, MeJA, and MT samples). The samples in the three groups were treated as follows: 10 freshly broccoli heads were used for the analysis on the day of harvest; 60 broccoli heads were sprayed evenly with distilled water; $600 \mu \mathrm{L}$ MeJA (SigmaAldrich, USA) standard (95\%) was dissolved in distilled water with the addition of $1 \%$ ethanol solution. Then, the MeJA solution $\left(0.5 \mu \mathrm{mol} \mathrm{L}{ }^{-1}\right)$ was used for spraying on the 60 broccoli heads; 60 samples were sprayed with the aqueous solution of melatonin (MT) (Sigma-Aldrich, USA) $\left(100 \mu \mathrm{mol} \mathrm{L}^{-1}\right)$. All the broccoli heads were dried uniformly after the treatments, and were subsequently used for the storage experiments.

The samples were packed in polyethylene bags (thickness: $0.03 \mathrm{~mm}$ ), with ten heads per bag, and the bags were sealed by folding. Finally, the samples were stored at $20{ }^{\circ} \mathrm{C}$ under $78-80 \%$ relative humidity. The test cycle was divided into two phases. In the first stage (day 0-4), the samples were tested once daily, whereas during the second stage (day 4-8), the samples were tested at intervals of two days. Besides, the samples of all treatments were taken on sampling time until the broccoli completely yellowed. All the measurements were performed restoring the samples to the room temperature. 3 replicates of 6 broccoli heads were taken for each sampling date and treatment, and the whole experiment was repeated three times.

\subsection{Analysis of the volatile compounds}

The methods described by Lv et al. ${ }^{14}$ were used for the extraction and determination of the broccoli volatiles. A sample of broccoli florets $(3.0 \mathrm{~g})$ was placed in a vial with $20 \mathrm{~mL}$ headspace and $2.5 \mathrm{~g} \mathrm{NaCl}$ and $10 \mu \mathrm{L}$ 2-nonanone internal standard $0.25 \mathrm{mg}$ $\mathrm{mL}^{-1}$ ) were added; the vial was sealed with a Teflon septum aluminum cap and the contents were mixed. For manual solidphase microextraction (SPME) analysis, the samples were equilibrated at $50{ }^{\circ} \mathrm{C}$ for $40 \mathrm{~min}$ and then exposed to a fiber coated with $65 \mu \mathrm{m}$ of polydimethylsiloxane (Supelco Co., Bellefonte, PDMS) at $50{ }^{\circ} \mathrm{C}$ for $40 \mathrm{~min}$. Subsequently, the volatiles were desorbed over $180 \mathrm{~s}$ at $250{ }^{\circ} \mathrm{C}$ into the splitless injection port. The volatiles were analyzed with a GC-MS apparatus (7890A-5975C, Agilent Technologies, USA) equipped with HPINNOWAX $\left(3 \times 10^{4} \mathrm{~mm}\right.$ length $\times 0.25 \mathrm{~mm}$ i.d. $\times 2.5 \times$ $10^{-4} \mathrm{~mm}$ film thickness) fused silica capillary column. The heating program was as follows: the injector was maintained at $250{ }^{\circ} \mathrm{C}$; the initial oven temperature was $40{ }^{\circ} \mathrm{C}$, which was held for $3 \mathrm{~min}$; the temperature then increased to $120{ }^{\circ} \mathrm{C}$ at $5{ }^{\circ} \mathrm{C} \min ^{-1}$, and was subsequently raised to $200{ }^{\circ} \mathrm{C}$ at $10{ }^{\circ} \mathrm{C} \min ^{-1}$ and held for $5 \mathrm{~min}$. The MS conditions were as follows: the temperature of the electron ionization source was maintained at $200{ }^{\circ} \mathrm{C}$ and the mass scanning was done in the 35 to $500 \mathrm{~m} / \mathrm{z}$ range. The flow rate of helium was $1.0 \mathrm{~mL} \mathrm{~min}^{-1}$. The NIST 11 spectra library was used to identify the volatile compounds. By comparing the linear retention indices and the EI mass spectra with those of the reference compounds, the identities of most of the compounds were confirmed. The compounds were quantified using the internal standard method, where the concentrations of various volatile components were normalized to that of 2-nonanone.

\subsection{Electronic tongue measurement}

The gustatory sensation during the storage process of broccoli in the different treatment groups was determined by using the electronic tongue (SA402B, INSENT, Japan). The first step was the self-examination and preparation of the electronic tongue system until the sensors (ZZ, BA, BB, CA, GA, HA, and JB) were rinsed with distilled water for several minutes to obtain constant readings using water and a reference electrode $(\mathrm{Ag} /$ AgCl $3 \mathrm{M} \mathrm{KCl}$ ) (Metrohm AG), immersed in the liquid sample. 
The tissue of broccoli florets (50 g) was ground and filtered through six layers of gauze; three biological replications were performed. Finally, $100 \mathrm{~mL}$ filtrate was injected into the measuring cup of the automatic sampler to start the experiment. The test parameters were set three times, and the sourness, saltiness, sweetness, bitterness, astringency, and umami tastes were tested.

\subsection{Determination of the content of bioactive compounds}

2.4.1 Determination of flavonoid content. Flavonoids were extracted from the broccoli. The $2.0 \mathrm{~g}$ tissue (florets) was triturated with $70 \%$ ethanol and placed in a water bath at $85{ }^{\circ} \mathrm{C}$ for $1.5 \mathrm{~h}^{20}$ And then the total flavonoid content was determined by the spectrophotometric method. $2 \mathrm{~mL}$ extract was mixed with $0.6 \mathrm{~mL}$ of $5 \% \mathrm{NaNO}_{2}$ solution and incubated for $5 \mathrm{~min}$ avoid light. And then the mixture was added with $0.6 \mathrm{~mL} 10 \%$ $\mathrm{Al}\left(\mathrm{NO}_{3}\right)_{3}$ for incubating $6 \mathrm{~min}$ in the dark. Add $4 \mathrm{~mL}$ of $4 \%$ $\mathrm{NaOH}$ solution to the mixture, dilute to $10 \mathrm{~mL}$ with deionized water, and place in the dark for $20 \mathrm{~min}$. Finally, the absorbance was measured by using spectrophotometer at $510 \mathrm{~nm}$.

The standard solution of quercetin in different concentrations is obtained by repeating the same procedure. The quercetin standard was obtained from DingGuo Changsheng Biotechnol CO. LTD (Beijing). The flavonoid content was calculated using the following calibration curve: $Y=-0.0134 X+$ 12.188, $R=0.9968$, where $X$ is the flavonoid content in $\mathrm{mg} \mathrm{g}^{-1}$ and $Y$ is the absorbance. The total flavonoid content was expressed as $\mathrm{mg}$ of quercetin equivalent/g of fresh weight (FW). Three biological replicates were used for each treatment, and the experiments were performed three times.

2.4.2 Determination of $\mathrm{V}_{\mathrm{C}}$ content. The content of $\mathrm{V}_{\mathrm{C}}$ was determined as described in a previous report ${ }^{21}$ and analyzed by high performance liquid chromatography-mass spectrometer (HPLC-MS). The ice-cold extraction solution consisted of 2 mmol L ${ }^{-1}$ disodium-dihydrogen-EDTA and 6\% meta-phosphoric acid. $5 \mathrm{~g}$ broccoli buds tissue was triturated with $5 \mathrm{~mL}$ ice-cold extraction solution, and the additional $5 \mathrm{~mL}$ solution was added in the extract. And then, the extract was homogenized for $2 \mathrm{~min}$ in the absence of light. The homogenate was filtered twice by filter paper, and the residue was washed with $5 \mathrm{~mL}$ ice-cold extraction solution. Then, the total homogenate was filtered again. Finally, determination solution consisted of $100 \mu \mathrm{L}$ filtrate and $400 \mu \mathrm{L} 0.87 \%$ ortho-phosphoric acid solution containing disodium-dihydrogen-EDTA $\left(1 \mathrm{mmol} \mathrm{L}^{-1}\right)$ and Trizma Base $\left(100 \mathrm{mmol} \mathrm{L}^{-1}\right)$, which was used for the determination of $\mathrm{V}_{\mathrm{C}}$ content.

The HPLC-MS analysis was carried out using the LCMS-8050 spectrometer (Shimadz, Kyoto, Japan). The flow rate was set at

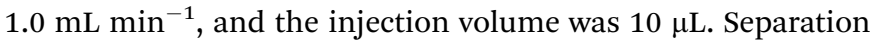
was performed on a $2 \mu \mathrm{m}$ Slum-pack GIS C18 column $(75 \mathrm{~mm} \times$ $2.1 \mathrm{~mm}$; Shimadz, Kyoto, Japan) at $25{ }^{\circ} \mathrm{C}$. The mobile-phase solvent system used was acetonitrile (2\%): $\mathrm{NaH}_{2} \mathrm{PO}_{4}(50 \mathrm{mmol}$ $\left.\mathrm{L}^{-1}\right)$ : dodecyltrimethyl ammonium chloride $\left(2.5 \mathrm{mmol} \mathrm{L}^{-1}\right)$ : disodiumdihydrogen-EDTA (1.25 $\left.\mathrm{mmol} \mathrm{L}^{-1}\right)(39 / 13 / 13 / 13$, by volume), and isocratic flow terminated each run. $\mathrm{V}_{\mathrm{C}}$ standards were obtained from DingGuo Changsheng Biotechnol CO.LTD
(Beijing, China). The quantification of $\mathrm{V}_{\mathrm{C}}$ was carried out by the standard calibration method and the results were expressed as $\mathrm{mg}$ of ascorbic acid/100 $\mathrm{g}$ of fresh weight (FW). The peak areas for $\mathrm{V}_{\mathrm{C}}$ were evaluated in triplicate for each treatment.

2.4.3 Extraction of carotenoids and HPLC-MS analysis. Carotenoids were extracted from broccoli florets as described previously and were analyzed by HPLC. ${ }^{22}$ The broccoli bud tissue was triturated with methanol/chloroform/water $(1: 2: 1$, $\mathrm{v} / \mathrm{v} / \mathrm{v}$ ), and the extract was centrifuged at $8000 \times g$ for $15 \mathrm{~min}$. The aqueous phases were extracted with chloroform several times until the residue was colorless. The residue was dried under a gentle stream of nitrogen. The reaction was terminated by addition of $6 \%(\mathrm{w} / \mathrm{v}) \mathrm{KOH}$ (in methanol) and incubation in a water bath at $60{ }^{\circ} \mathrm{C}$ for $30 \mathrm{~min}$ in the absence of light. The dried residues were re-suspended with the nitrogen stream and applied to the HPLC-MS.

The HPLC-MS analysis was carried out using the liquid chromatograph mass spectrometer (LCMS-8050, Shimadz, Kyoto, Japan). The injection volume was $10 \mu \mathrm{L}$, and flow rate was set to $0.2 \mathrm{~mL} \mathrm{~min}^{-1}$. Separation was performed on a $2 \mu \mathrm{m} \times$ $75 \mathrm{~mm} \times 2.1 \mathrm{~mm}$ column (Slum-pack GIS C18; Shimadz, Kyoto, Japan) and operated at $40{ }^{\circ} \mathrm{C}$. The mobile-phase solvent system used was acetonitrile: methanol ( $0.1 \mathrm{M}$ ammonium formate): dichloromethane: water (65/20/6/9, by volume), and isocratic flow terminated each run. After equilibrating the column, the carotenoids contained (zeaxanthin, $\beta$-cryptoxanthin and $\beta$ carotene) in the samples were separated and quantified using the standard calibration curve. The standards were obtained from DingGuo Changsheng Biotechnol CO. LTD. (Beijing, China). Three biological replicates were used for each treatment, and the experiments were performed three times. The sum of zeaxanthin, $\beta$-cryptoxanthin and $\beta$-carotene content was taken as the carotenoids content in broccoli.

2.4.4 Determination of sulforaphane content. Sulforaphane was extracted according to the method described in a previous report. ${ }^{23}$ The floret tissue $(0.2 \mathrm{~g})$ was milled in liquid nitrogen using a pre-cooled mortar and mixed with $2 \mathrm{~mL}$ of 0.5 $\mathrm{U} \mathrm{mL}^{-1}$ myrosinase solution. Acetonitrile $(1 \mathrm{~mL})$ was added to the liquid for dissolving the residue and the homogenate was passed through a PTFE $(0.45 \mu \mathrm{m})$ membrane syringe filter before injecting into the HPLC. The samples were separated at $30{ }^{\circ} \mathrm{C}$ on a Kromasil $\mathrm{C} 18$ column $(250 \times 4.6 \mathrm{~mm})$ using acetonitrile and water at a flow rate of $1.0 \mathrm{~mL} \mathrm{~min}^{-1}$. Sulforaphane (Sigma) was used as an external standard for HPLC analysis. The absorbance was measured at $254 \mathrm{~nm}$.

2.4.5 Measurement of antioxidant activity. The antioxidant activity was measured in terms of DPPH radical scavenging activity, which was determined using the modified described by Hatano et al. ${ }^{24}$ after some modification. The DPPH standard was dissolved in anhydrous ethanol. The initial concentration of DPPH was $10 \times 10^{-5} \mathrm{~mol} \mathrm{~L}^{-1}$, which was diluted into solution of five different concentrations. A calibration curve was drawn by measuring the absorbance of the standard solutions at $765 \mathrm{~nm}$ using a UV spectrophotometer. The DPPH standard (0.2 g) was dissolved in anhydrous ethanol and a $2 \times 10^{-4} \mathrm{~mol} \mathrm{~L}^{-1}$ DPPH diluent was prepared. Tissue from broccoli buds $(5.0 \mathrm{~g})$ was milled in $50 \%$ ethanol solution $(50 \mathrm{~mL})$ and the extract was 
centrifuged at $8000 \times g$ for $10 \mathrm{~min}$. The following reaction fluid was added to the extract: A ( $2 \mathrm{~mL}$ DPPH and $2 \mathrm{~mL}$ anhydrous alcohol), $\mathrm{A}_{0}$ (anhydrous alcohol), B (2 mL DPPH and $2 \mathrm{~mL}$ sample solution), and $\mathrm{B}_{0}(2 \mathrm{~mL}$ anhydrous alcohol and $2 \mathrm{~mL}$ sample solution); the absorbance was measured at $765 \mathrm{~nm}$. Finally, the absorbance value was converted to the molar concentration of DPPH according to the standard curve. The results were expressed as milligram of gallic acid equivalent per gram fresh weight. The formula used for the calculation of $\mathrm{DPPH}$ radical scavenging activity was as follows:

$$
C_{1}=\left(1-\mathrm{C}_{\mathrm{DPPH}} / C_{0}\right) \times 100 \%
$$

$C_{1}(\%)$ is the DPPH radical scavenging active, $C_{\mathrm{DPPH}}\left(\mathrm{mol} \mathrm{L}^{-1}\right)$ is the absorbance of the sample, and $C_{0}\left(\mathrm{~mol} \mathrm{~L}^{-1}\right)$ is the initial concentration of DPPH.

\subsection{Data analysis}

The results of electronic tongue measurements were evaluated by multivariate pattern recognition algorithms with the help of Statistica v. 9.0 software (StatSoft, Inc., Tulsa, OK). Data are presented as means \pm standard deviation of three replicates and were analyzed using SPSS v.20 software (SPSS Inc., Chicago, IL, USA). Differences among the groups were evaluated by oneway analysis of variance, and the relationship between the indices was analyzed by Pearson's correlation. The figures were prepared using Prism 5.0 software (Graph Pad Inc., La Jolla, CA, USA).

\section{Results}

\subsection{Effect of different treatments on the color of broccoli}

The color change is an important sensory index for broccoli that intuitively reflects the effect of post-harvest handling. As shown in Fig. 1, broccoli heads began to turn yellow on the second day of harvest, when stored at $20{ }^{\circ} \mathrm{C}$ without any treatment. In contrast, the color of broccoli heads treated with MeJA and MT barely showed any change. On the fourth day of the shelf life, the control samples were so severely yellowed that they had no commercial value, whereas the MeJA-treated samples showed slight signs of yellowing and the color of broccoli heads treated with MT remained bright green. Compared to that in the control samples, in the MeJA and MT treated samples, the yellowing process of broccoli heads was effectively delayed by 2 and 4 days, respectively. Based on subsequent studies, it was clear that the control, MeJA, and MT samples turned yellow on day 2,4 , and 6 , respectively.

\subsection{Effects of different treatments on the volatile components}

As shown in Table 1, only three kinds of sulfurous volatile compounds were detected in fresh samples, namely hexanal, $(E)$ 2-hexenal, and low levels of volatile alcohols and esters. Compared to the fresh samples, the sulfides were unchanged when the control and MeJA samples turned yellow, whereas those in the MT-treated product increased from three to five. The

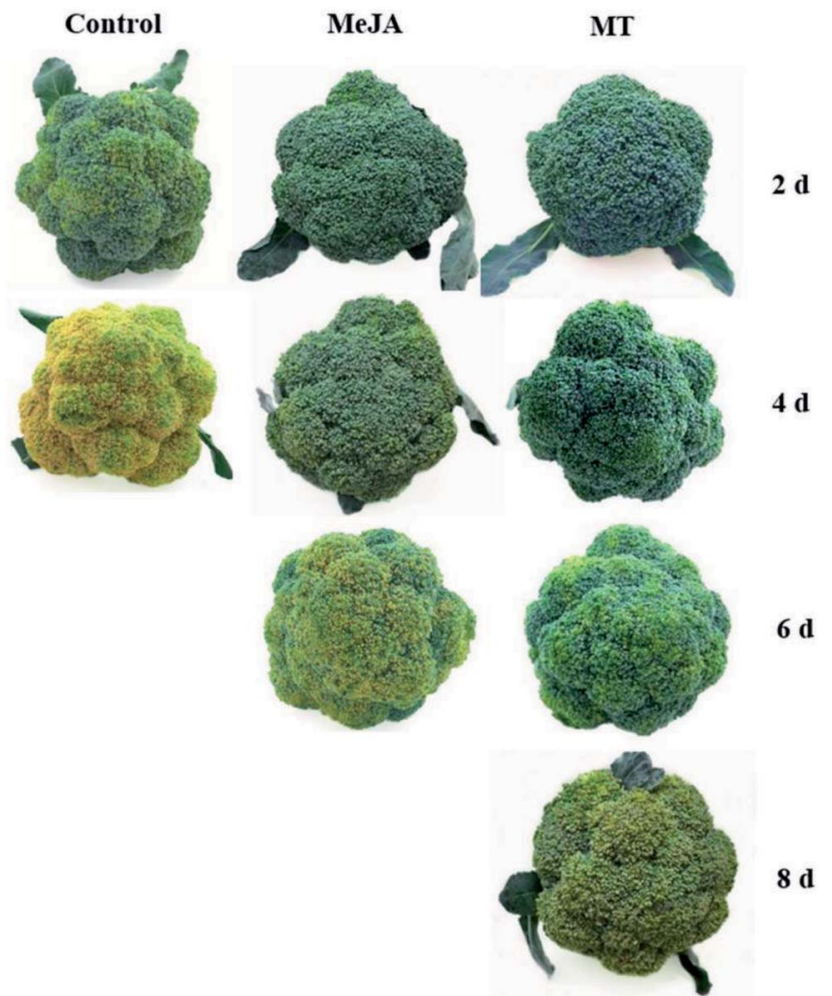

Fig. 1 Color changes in broccoli on day 2, 4, 6, and 8 in the different treatment groups, viz. control, 3 methyl jasmonate (MeJA), and melatonin (MT).

content of sulfides in the control was $13.2 \%$ lower than that in the fresh samples, whereas that in the MeJA and MT-treated samples was significantly decreased and increased $(P<0.05)$ by $72.9 \%$ and $40.9 \%$, respectively, compared to their content in the control samples. Moreover, no significant difference in the composition of sulfide compounds was found between the control and fresh samples. In the MeJA-treated samples, dimethyl disulfide and dimethyl trisulfide were the main components whose contents were changed with respect to those in the control samples, whereas methyl disulfide was a unique sulfide in the MT-treated samples when broccoli began to turn yellow.

In addition to sulfides, nine kinds of esters were detected in broccoli, including ethenyl hexanoate, $(Z)$-3-hexen-1-ol acetate, ethyl acetate, (Z)-3-hexen-1-ol propanoate, cis-3-hexenyl isobutyrate, isothiocyanates, butyl isothiocyanate, $N$-valeric acid cis-3-hexenyl ester, and 2-methylbutyl isothiocyanate. Six (control), five (MeJA), and three (MT) different kinds of volatile esters were detected on the day of turning yellow, while the day of harvesting was five. Unlike for the fruity plant, the abundance of esters in broccoli was not high, although broccoli had a rich variety of esters. When broccoli buds turned yellow from green, there was almost no difference in the kind and content of volatile esters $(P<0.05)$ in the control samples. However, compared to the control samples, the MT treatment caused a $12.8 \%$ drop in the content of volatile esters, whereas no significant change $(P<0.05)$ was observed in the MeJA-treated samples. Cis-3-hexenyl iso-butyrate only existed in the fresh samples, whereas 2-methylbutyl isothiocyanate was detected in 
Table 1 Changes of the volatile compounds in control, methyl jasmonate (MeJA), and melatonin (MT) samples on the day of harvesting and turning yellow ${ }^{a}$

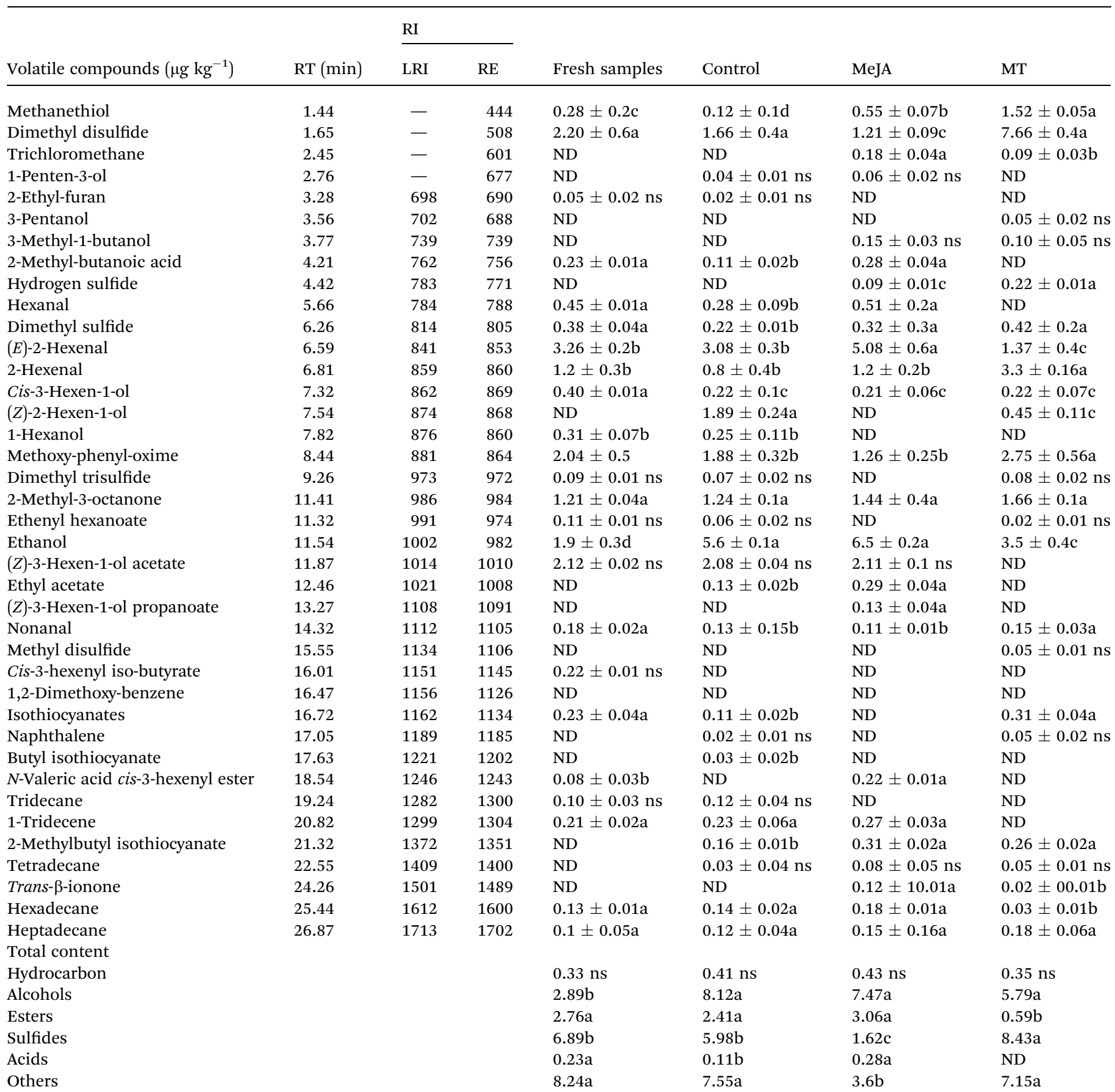

${ }^{a}$ RT and RI are the retention time and retention index of the volatile compounds, respectively. RE is the abbreviation for reference. The analyses were identified by comparison with the NIST 11 database. The symbol a, b, c, and d show significant differences according to the independent sample $t$-test $(P<0.05)$ for each volatile compound. 'ns' indicates no significant difference. ND indicates that the volatile compound was not detected. '- ' indicates that the retention time of this volatile compound is before the retention time of C6 $n$-alkanes.

all the yellowed samples. Butyl isothiocyanate and (Z)-3-hexen1-ol acetate were the unique volatile esters in the control and MT samples, respectively. In the MeJA samples, (Z)-3-hexen-1-ol propanoate was a unique volatile ester component. Furthermore, we found that the different treatments had little effect on the contents of hydrocarbons, alcohols, and aldehydes in broccoli.

\subsection{Effect of different treatments on the taste}

As shown in Table 2, when the control samples began to turn yellow, the level of sweetness and bitterness increased by $446.1 \%$ and $349.4 \%$, respectively, whereas there was no significant difference $(P<0.05)$ in the other flavors. When the samples turned yellow from green, compared to the control samples, the sourness and sweetness of the MeJA-treated 
Table 2 Effects of different treatments on broccoli flavor on the day of harvesting and turning yellow ${ }^{a}$

\begin{tabular}{lcccc}
\hline & \multicolumn{2}{l}{ Treatments } & & \\
\cline { 2 - 5 } Flavor compounds & Fresh samples & Control & MeJA & MT \\
\hline Sweetness & $-2.39 \pm 0.23 \mathrm{c}$ & $8.27 \pm 0.04 \mathrm{~b}$ & $17.39 \pm 0.11 \mathrm{a}$ & $-2.03 \pm 0.04 \mathrm{c}$ \\
Sourness & $-46.38 \pm 0.15 \mathrm{~b}$ & $-44.29 \pm 0.08 \mathrm{~b}$ & $-23.44 \pm 0.14 \mathrm{a}$ & $-45.24 \pm 0.15 \mathrm{~b}$ \\
Saltiness & $31.02 \pm 0.27 \mathrm{~ns}$ & $25.12 \pm 0.11 \mathrm{~ns}$ & $31.22 \pm 0.18 \mathrm{~ns}$ & $30.66 \pm 0.27 \mathrm{~ns}$ \\
Bitterness & $-0.81 \pm 0.11 \mathrm{c}$ & $2.02 \pm 0.22 \mathrm{~b}$ & $-12.2 \pm 0.02 \mathrm{~d}$ & $8.32 \pm 0.07 \mathrm{a}$ \\
Astringency & $1.02 \pm 0.03 \mathrm{~b}$ & $1.74 \pm 0.07 \mathrm{~b}$ & $0.22 \pm 0.10 \mathrm{~b}$ & $7.29 \pm 0.08 \mathrm{a}$ \\
Umami & $14.23 \pm 0.23 \mathrm{~b}$ & $14.91 \pm 0.02 \mathrm{~b}$ & $12.4 \pm 0.09 \mathrm{~b}$ & $31.33 \pm 0.07 \mathrm{a}$
\end{tabular}

${ }^{a}$ Values represent the means \pm standard deviation of three replicates of six broccoli heads $(n=9)$. Different letters in the different rows indicate significant differences under different treatments $(P<0.05)$. 'ns' indicates no significant difference.

Table 3 Effects of different treatments on the contents of different bioactive compounds in broccoli and on the antioxidant activity on the day of harvesting and turning yellow ${ }^{a}$

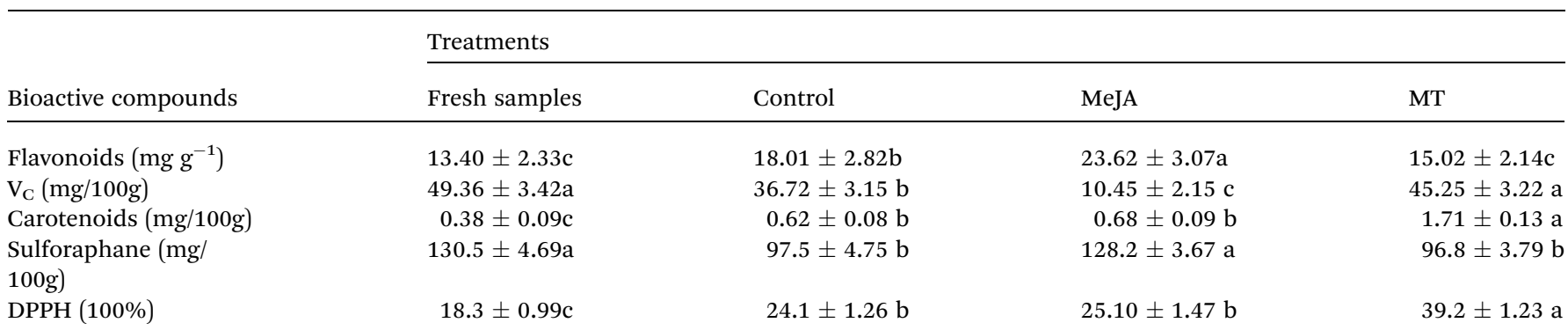

${ }^{a}$ Values represent the means \pm standard deviation of three replicates of six broccoli heads $(n=9)$. Different letters in different rows indicate significant differences under different treatments $(P<0.05)$. 'ns' indicates no significant difference.

samples increased by $47.1 \%$ and $110.3 \%$, whereas the bitterness was significantly decreased $(P<0.05)$ by $710.2 \%$. For the MT samples, the level of bitterness, astringency, and umami increased by about 50.5\%, 319.1\%, and $110.1 \%$, respectively, compared to that in the samples without any treatment, whereas the sweetness was significantly decreased $(P<0.05)$ by $124.2 \%$.

\subsection{Effect of different treatments on the bioactive compounds}

Broccoli is a good source of bioactive compounds with antioxidant, immuno-regulatory, and anti-aging effects. We detected the content of flavonoids, $\mathrm{V}_{\mathrm{C}}$, carotenoids, and sulforaphane in broccoli heads on the day of harvesting and turning yellow after storage at $20^{\circ} \mathrm{C}$. As shown in Table 3, when the control samples changed from green to yellow, the sulforaphane and $\mathrm{V}_{\mathrm{C}}$ content decreased by $25.3 \%$ and $25.6 \%$, whereas the contents of flavonoids and carotenoids increased by $34.4 \%$ and $63.2 \%$. Compared to the control samples, MeJA treatment significantly reduced $(P<0.05)$ the $\mathrm{V}_{\mathrm{C}}$ content by $71.5 \%$, and increased the sulforaphane and flavonoids content by $31.5 \%$ and $30.5 \%$. The contents of $\mathrm{V}_{\mathrm{C}}$ and carotenoids in the MT-treated samples was significantly increased $(P<0.05)$ by $23.2 \%$ and $175.8 \%$, respectively whereas those of other bioactive compounds tested in this study were not significantly different from the control samples. We also observed that the MT treatment prevented the decrease of $\mathrm{V}_{\mathrm{C}}$ and carotenoids, but not of the flavonoids.
Moreover, in the MeJA treated samples, the content of sulforaphane and flavonoids was significantly accumulated $(P<0.05)$, but the normal level of $\mathrm{V}_{\mathrm{C}}$ could not be maintained.

\subsection{Effect of different treatments on the antioxidant activity}

The DPPH radical scavenging activity is used to measure the antioxidant ability in plants. As shown in Table 3, compared to the fresh samples after harvest, the DPPH radical scavenging activity of all the samples was increased gradually accompanied by the yellowing of broccoli heads. These results were in agreement with those of other studies on broccoli. ${ }^{25}$ Moreover, the DPPH in the control samples was significantly higher than that in the fresh samples $(P<0.05)$, and was about $62.7 \%$ lower than that in the MT samples. There was no significant difference $(P<0.05)$ in DPPH between the control and MeJA samples when the buds began to turn yellow.

\section{Discussion}

Sensory quality, including color, aroma, flavor, and tissue morphology, is one of the important factors for customers in choosing fruits and vegetables. The broccoli heads are composed of tightly clustered buds. The freshly picked broccoli samples were green, whereas the buds stored at $20{ }^{\circ} \mathrm{C}$ without treatment began to turn yellow on day 2 during the shelf life. During the storage, the permeability of different cell membranes would change, resulting in the disintegration of the 
chloroplast membrane and deformation of the thylakoid membrane structure. The chlorophyll attached to the thylakoid membrane would thus be released and consumed by extensive action of the chlorophyll degrading enzymes. Moreover, because of the transformation of plastids, chloroplasts and leucoplasts are converted to chromoplasts, which store a large number of carotenoids. In this way, while the green pigment (chlorophyll) is consumed, a large amount of yellow pigment (carotenoids) accumulates, which in turn causes the yellowing of broccoli. ${ }^{26}$ It has previously been shown that the yellowing of plants was closely related to reactive oxygen species and cell membrane components. ${ }^{27}$

In this study, the MeJA-treated broccoli heads maintained a high level of sensory quality on day 4, whereas the control samples turned completely yellow. Previous studies have suggested that MeJA induced the production of ethylene to accelerate the yellowing process. However, the effect of MeJA treatment is related to factors such as treatment concentration and the maturity of fruits and vegetables, and this view has been confirmed in the relevant research results of apple quality. ${ }^{28}$ Simultaneously, MeJA treatment could maintain the surface color of the fruit while reducing the rate of quality change during storage. Feng et al. ${ }^{29}$ found that MeJA treatment could prevent the mango chilling without accelerating coloration. Dong and $\mathrm{Cai}^{30}$ proved that MeJA application significantly increased chlorophyll content, chlorophyll fluorescence parameter $F_{\mathrm{v}} / F_{\mathrm{m}}$ and $F_{\mathrm{v}} / F_{0}$ in rice leaves under drought conditions. Moreover, the slightly delayed yellowing process in the MeJA treatment might be due to the enhanced adaptation of broccoli under the unsuitable storage temperature used in the study. MeJA plays an important role as a signal molecule in plant-induced responses and defense mechanisms. Evidence ${ }^{31}$ suggests that when plants are under stress, MeJA rapidly accumulated to a high content, in response to the adversity, and thereby, participating in and regulating many physiological and biochemical processes of the plant for improving the resistance to adversity. The functions of MT, including its antioxidant, anti-inflammatory properties, and the capacity to modulate mitochondrial homeostasis, are linked to the redox status of cells and tissues. MT is important for the physiological regulation of cellular homeostasis. ${ }^{32}$ In addition, Szafrańska et al. ${ }^{33}$ studied the ultrastructure of Vigna Radiata roots and found that melatonin effectively protected the structure of plastids (chloroplast, chromoplast, leucoplast), which could prove that MT was helpful in maintaining the morphology of the chloroplast and thylakoid membranes. Thus, the yellowing process of the MT-treated broccoli samples was remarkably delayed.

Aroma is an important index that reflects the flavor, maturity, and commodity quality of fruits and vegetables. The pleasant aroma is an important factor in attracting the consumers and in enhancing the market competitiveness of the product. Aroma is determined by the content, composition, and odor threshold of the different volatile components. Broccoli is characterized by sulfurous volatile compounds, which are similar to that in other cruciferous plants. Furthermore, esters and aldehydes provide fruity and grassy aroma, respectively, whereas alcohols are often accompanied with the spoiling odors. Dimethyl disulfide, disulfide dimethyl, dimethyl trisulfide, hexanal, cis-3-hexen-1-ol, and ethanol acetate were identified as the main volatile components in broccoli (Table 1). This result was similar to that obtained by Annelie et al. ${ }^{34}$ in broccoli. As per our findings, sulfides are the main volatile components of cruciferous vegetables. The GC-MS analysis revealed that there was no significant change in the content of sulfides when the untreated sample was yellowed, which might be because of the short storage time during which the change in total sulfide content could not be registered. The treatment of broccoli samples with MeJA could significantly reduce the unique sulfide volatiles, allowing broccoli to maintain fresh aroma even when they started to turn yellow. This might be due to the differences in the levels of dimethyl disulfide in the MeJA-treated samples. The MT treatment increased the production of volatile sulfides in the yellowed broccoli and was accompanied with a fullbodied odor because of deterioration of the lipid membranes in the cells as well as because of the loss of intracellular compartmentalization due to prolonged storage, which resulted in enzymatic reactions. ${ }^{35}$ In addition, MT might promote the accumulation of the free amino acid, $S$-methyl-L-cysteine sulfide, which is the main source of volatile sulfide compounds.

When the control samples turned yellow, the types of aldehydes and esters as well as their contents were not significantly changed. In the MeJA-treated samples, when the buds just lost their green color, the content of $(E)$-2-hexenal, which is used to evaluate the volatile content of plants, was increased. It might be that MeJA treatment promoted the accumulation of aldehydes, ${ }^{36}$ which provided fresh grass aroma to broccoli. In addition, 2-hexenal was the main component of leaf volatiles, which had been effectively increased by MT treatment during yellowing. MT induced the synthesis of the precursor substances of 2-hexenal volatile compounds, which were mainly formed by the aldol condensation pathway. ${ }^{37}$ Moreover, the MT treatment resulted in a sharp decrease in the ester content of the yellowing samples, which was different from that in the control and MeJA samples. This might be related to the reduction in the types of ester present in such samples.

A survey conducted by the Regional Fruit and Vegetables Economic Committee found evidence that flavor is one of the main reasons for some consumers rarely or never purchasing cauliflower. ${ }^{38}$ Some consumers were very sensitive to the unique sulfide taste of Brassica vegetables, which gives a pungent odor and bitter taste to these vegetables. ${ }^{12}$ Unlike other taste analysis instruments used throughout the world, the electronic tongue has a real taste analysis system, which matches the tastes in humans. ${ }^{39}$ In our study, we detected the changes in sweetness, sourness, saltiness, bitterness, astringency, and umami of broccoli heads during yellowing and assessed the effects of different treatments on these changes. The results were obtained by an electronic tongue detector in the form of unitless numerical values, where a larger number indicated a more intense flavor. We found that the untreated samples had a large increase in sweetness and bitterness when they began to turn yellow. This might be related to the accumulation of carbohydrates in the broccoli samples after the harvest. At this time, the flavor of broccoli was better than that at the time of harvest. 
Different treatments have influence on the specific flavor of Brassica vegetables, because of the enzymatic reaction products which play the significant roles in the creation of flavors. ${ }^{40}$ Previous studies have shown that the products of hydrolysis of glucosinolates play a key role in determining the flavor of Brassica vegetables. ${ }^{\mathbf{4 1}}$ The relationship between myrosinase and the feeling of taste was investigated by Engel et al. ${ }^{38}$ They found that the products of the hydrolysis of glucosinolates could explain the feeling of bitterness. In our study, a high level of sulforaphane was observed in the MeJA samples when they began to turn yellow. As a hydrolysate of glucosinolates, sulforaphane affect the binding of bitter taste receptors to precursor substances, thereby, reducing the bitterness. On the other hand, MeJA effectively induced the accumulation of sugars and increased the sweetness. ${ }^{42}$ The decrease in bitterness and the added sweetness resulted in broccoli having a better flavor when they began to turn yellow. These findings were especially interesting considering the interactions of taste molecules and potential masking of bitterness by the sweet taste. The complex role of isothiocyanates in the perception of flavor of Brassica vegetables was attributed to the interaction with bitterness taste receptors. ${ }^{43}$ The content of isothiocyanates in the MT-treated samples was higher than that in the MeJA samples, which provided the material basis for bitterness receptors, and increased the bitter flavor of the yellowed samples. As a flavor compound, isothiocyanates also contributed to the aroma of broccoli. Moreover, the improvement in the bitterness, astringency, and umami of broccoli samples treated with MT might be related to the high content of volatile sulfides when broccoli turned yellow from green. The above contents confirmed the close relationship between the volatile components and flavor.

Broccoli is rich in various nutrients, which is one of the important factors that attract consumers. Flavonoids and $\mathrm{V}_{\mathrm{C}}$ have indisputable antioxidant, and cancer and chronic disease fighting properties, and carotenoids are the main source of vitamin A in the body. We found that even if the control samples started to turn yellow, the contents of flavonoids, and carotenoids continued to increase because of the shorter storage times. There are large amounts of oxidases in broccoli and we inferred that it might be due to an increase in the oxidase activity during the yellowing process, resulting in different degrees of loss of $\mathrm{V}_{\mathrm{C}}$ content in the MeJA samples. However, when the MT-treated broccoli samples began to turn yellow, we observed increased levels of carotenoids and $\mathrm{V}_{\mathrm{C}}$ due to the effective inhibition of oxidation and scavenging of free radicals by MT. Besides, the effect of MT was indirectly demonstrated by the increase in DPPH in the broccoli samples, accompanying the yellowing process. In our study, flavonoids were not affected by MeJA and MT. It might be possible that the signal transduction and free radicals do not directly participate in the biosynthetic pathway of flavonoids.

Sulforaphane is an important secondary metabolite in broccoli, which is widely known for its superior antioxidant properties. When broccoli lost the green color, the sulforaphane level in the control samples was drastically reduced. The reduction in sulforaphane content might be a result of environmental discomfort and the depletion of glucosinolates. Pérez-Balibrea et al. ${ }^{44}$ found that the synthesis and content of sulforaphane were directly influenced by the exogenous treatments. MeJA stimulated the production of secondary metabolites in many plant species. In our study, the sulforaphane level in the MeJA-treated samples was significantly higher $(P<0.05)$ than that in the control samples. MeJA induced the synthesis of glucosinolates and affected the content of sulforaphane in broccoli. It significantly improved the sulforaphane content, as was also reported by Dombrecht. ${ }^{45}$ In addition, Halkier and Gershenzon $^{46}$ indicated that MeJA stimulated the biosynthesis of indoles, which was due to the anabolic effect of jasmonate compounds and aliphatic amino acids, and effected the accumulation of sulforaphane. However, MT did not have a similar effect, showing no difference in the sulforaphane content when compared to the control samples of yellowed broccoli.

MeJA was first discovered and isolated from the essential oil of Jasminum grandiflorum L. by Demole et al.,${ }^{47}$ which was a flavor compound. Thus, the source of MeJA is safer than other plant hormones. The significance of MeJA for higher plant metabolism is beyond doubt, and it also has a positive contribution to human health. A large number of reports indicate that MeJA has an anti-cancer effect in the clinic, and the effect was significant. ${ }^{\mathbf{4 8 , 4 9}}$ Kniazhanski et al. ${ }^{50}$ found that MeJA was effective against cervical carcinoma cell lines. Furthermore, MeJA is the involvement of ROS in MeJA-induced apoptosis, which is the anti-cancer mechanism. ${ }^{51}$ In our study, the MeJA content in treatment $\left(0.5 \mu \mathrm{mol} \mathrm{L}{ }^{-1}\right)$ was extremely trace, which not only ensured food safety, but also increased the commercial value of anti-cancer in broccoli to some extent. MT is an indole hormone secreted by the pineal gland, which physiological functions include inducing sleep, regulating the endocrine system, maintaining stable internal environment, enhancing immunity, anti-aging and so on. ${ }^{52}$ MT mediated by specific receptors, which regulates the biological rhythm of sleep awakening and plays a role in sedation and induces sleep, so that it is called physiological hypnotic agent. ${ }^{53}$ In the regulation of melatonin, it has obvious therapeutic effects on sleep disorders which caused by sleep delay syndrome and abnormal time difference. Arendt et al. ${ }^{54}$ analyzed a number of clinical findings and found that the recommended melatonin intake was $0.5-5 \mathrm{mg}$ for each time. The concentration of MT used in our study was $100 \mu \mathrm{mol} \mathrm{L}^{-1}$, and the MT content was much lower than the daily recommended limit. Therefore, normal intake of broccoli would not affect the body.

\section{Conclusions}

In our study, the buds of untreated broccoli began to turn yellow on day 2 when stored at $20{ }^{\circ} \mathrm{C}$. The MeJA and MT treatments effectively delayed the yellowing time of broccoli by 2 and 4 days, respectively. When the control samples began to lose their green color, the substantial increase in sweetness and bitterness was accompanied by an increase in various bioactive compounds, except for sulforaphane; however, there was no significant change in the content of volatile components. Compared to the control samples, the MeJA treatment mainly 
contributed to the accumulation of sulforaphane and flavonoids, to sweetness and sourness, and to the relief from the special odor of sulfides. However, the MT treatment tended to improve the antioxidant activity and the increase of bitterness, astringency, umami, and volatile sulfides. These results showed that the flavor, aroma, and nutrients of broccoli that began to turn yellow was changed to some extent, and the MeJA and MT treatment could significantly regulate the content of bioactive compounds while enhancing the sensory quality. In addition, we believe that maintenance of the original flavor of fruits and vegetables should no longer be our main focus. We should continue to explore strategies to improve the distasteful flavor of fruits and vegetables. The MeJA and MT treatments can contribute to the improvement of flavor in broccoli, whatever its form might be.

\section{Conflicts of interest}

There are no conflicts to declare.

\section{Acknowledgements}

This work was supported by the National Key R\&D Program of China (no. 2016YFD0400103).

\section{References}

1 R. B. Jones, C. L. Frisina, S. Winkler, M. Imsic and R. B. Tomkins, Food Chem., 2010, 123, 237-242.

2 G. Yuan, B. Sun, J. Yuan and Q. Wang, Food Chem., 2010, 118, 774-781.

3 R. R. Dedolph, S. H. Wittwer, V. Tuli and D. Gilbart, Plant Physiol., 1962, 37, 509-512.

4 K. M. Ku, E. H. Jeffery and J. A. Juvik, J. Sci. Food Agric., 2014, 94, 2090-2096.

5 C. Wasternack and B. Hause, Ann. Bot., 2013, 111, 1021-1058.

6 M. K. Kang, E. H. Jeffery and J. A. Juvik, J. Agric. Food Chem., 2013, 61, 9623-9631.

7 C. Rodriguez, J. C. Mayo, R. M. Sainz, I. Antolín, F. Herrera and V. Martín, J. Pineal Res., 2004, 36, 1-9.

8 S. Park, D. Lee, H. Jang, Y. Byeon, Y. Kim and K. Back, J. Pineal Res., 2013, 54, 258-263.

9 M. M. Posmyk, M. Baå'Abusta, M. Wieczorek, E. Sliwinska and K. M. Janas, J. Pineal Res., 2009, 46, 214-223.

10 M. M. Posmyk, H. Kuran, K. Marciniak and K. M. Janas, J. Pineal Res., 2010, 45, 24-31.

11 M. N. Wieczorek, M. Walczak, M. Skrzypczak-Zielińska and H. H. Jeleń, Crit. Rev. Food Sci. Nutr., 2017, 56, 100-145.

12 J. Lv, J. Wu, J. Zuo, L. Fan, J. Shi and L. Gao, Food Chem., 2017, 216, 225-233.

13 V. Valpuesta and M. A. Botella, Trends Plant Sci., 2004, 9, 573-577.

14 M. V. Eberhardt, Y. L. Chang and H. L. Rui, Nature, 2000, 405, 903-948.

15 M. V. Eberhardt, K. Kobira, A. S. Keck, J. A. Juvik and E. H. Jeffery, J. Agric. Food Chem., 2005, 53, 7421.
16 Y. Topcu, A. Dogan, Z. Kasimoglu, H. Sahin-Nadeem, E. Polat and M. Erkan, Plant Physiol. Biochem., 2015, 93, 56-65.

17 R. Molaykumar, J. Lekhraj, I. Seiichiro and T. Tojiro, Food Chem., 2009, 114, 263-269.

18 F. Xu, X. Chen, J. Peng, X. Wang, J. Wang and Y. Zheng, Eur. Food Res. Technol., 2012, 235, 793-800.

19 J. J. Gills, E. H. Jeffery, N. V. Matusheski, R. C. Moon, D. D. Lantvit and J. M. Pezzuto, Cancer Lett., 2006, 236, 7279.

20 A. GliszczyńskaSwigło, E. Ciska, K. PawlakLemańska, J. Chmielewski, T. Borkowski and B. Tyrakowska, Food Addit. Contam., 2006, 23, 1088-1098.

21 A. Rybarczyk-Plonska, M. K. Hansen, A. B. Wold, S. F. Hagen, G. I. A. Borge and G. B. Bengtsson, Postharvest Biol. Biotechnol., 2014, 98, 82-89.

22 P. Stepnowski, K. H. Blotevogel and B. Jastorff, Int. Biodeterior. Biodegrad., 2004, 53, 127-132.

23 H. Liang, Q. P. Yuan, H. R. Dong and Y. M. Liu, J. Food Compos. Anal., 2006, 19, 473-476.

24 T. Hatano, H. Kagawa, T. Yasuhara and T. Okuda, Chem. Pharm. Bull., 1988, 36, 2090-20134.

25 P. Jin, D. Yao, F. Xu, H. Wang and Y. Zheng, Food Chem., 2015, 172, 705-709.

26 M. S. Tian, J. Am. Soc. Hortic. Sci., 1994, 119, 276-281.

27 J. D. Miller, R. N. Arteca and E. J. Pell, Plant Physiol., 1999, 120, 1015-1024.

28 X. Fan, J. P. Mattheis and J. K. Fellman, J. Am. Soc. Hortic. Sci., 1998, 123, 421-425.

29 F. Lei, Y. Zheng, Y. Zhang, F. Wang, L. Zhang and L. Zhaoxin, J. Agric. Food Chem., 2000, 48, 515-519.

30 T. Dong and K. Cai, Environ. Sci. Technol., 2009, 14, 156-187.

31 J. J. Cheong and Y. D. Choi, Trends Genet., 2003, 19, 409-413.

32 Y. Aguilera, T. Herrera, V. Benítez, S. M. Arribas, L. D. P. Al and R. M. Esteban, Food Chem., 2015, 170, 203-211.

33 K. Szafrańska, S. Glińska and K. M. Janas, Biol. Plant., 2012, 57, 91-96.

34 A. Jacobsson, A. Tim Nielsen and I. Sjöholm, J. Agric. Food Chem., 2004, 52, 1607-1614.

35 D. Zhao, J. Tang and X. Ding, LWT-Food Sci. Technol., 2007, 40, 439-447.

36 M. Yu, L. Shen, B. Fan, D. Zhao, Y. Zheng and J. Sheng, Postharvest Biol. Biotechnol., 2009, 54, 153-158.

37 E. Ortner and M. Granvogl, J. Agric. Food Chem., 2017, 41, 221-261.

38 E. Engel, C. Baty, D. Le Corre, I. Souchon and N. Martin, J. Agric. Food Chem., 2002, 50, 6459-6467.

39 A. Rudnitskaya, L. M. Schmidtke, A. Reis, M. R. Domingues, I. Delgadillo and B. Debus, Food Chem., 2017, 229, 20-27.

40 F. S. Hanschen, R. Klopsch, T. Oliviero, M. Schreiner, R. Verkerk and M. Dekker, Sci. Rep., 2017, 56, 712-761.

41 R. Kubec, A. Veronika Drhová and J. Velíšek, J. Agric. Food Chem., 1998, 46, 4334-4340.

42 L. Yu, Postharvest Biol. Biotechnol., 2016, 113, 8-16.

43 D. Zabaras, M. Roohani, R. Krishnamurthy, M. Cochet and C. Delahunty, Food Funct., 2013, 4, 592-601. 
44 S. Pérez-Balibrea, D. A. Moreno and C. García-Viguera, Food Chem., 2011, 125, 348-354.

45 B. Dombrecht, G. P. Xue, S. J. Sprague, J. A. Kirkegaard, J. J. Ross and J. B. Reid, Plant Cell, 2007, 19, 2225-2245.

46 B. A. Halkier and J. Gershenzon, Annu. Rev. Plant Biol., 2006, 57, 303-333.

47 E. Demole, E. Lederer and D. Mercier, Helv. Chim. Acta, 1962, 45, 512-567.

48 S. Cohen and E. Flescher, Phytochemistry, 2009, 70, 16001609.
49 E. Flescher, Cancer Lett., 2007, 245, 1-10.

50 T. Kniazhanski, A. Jackman, A. Heyfets, P. Gonen, E. Flescher and L. Sherman, Cancer Lett., 2008, 271, 34-46.

51 N. Goldin, A. Heyfets, D. Reischer and E. Flescher, J. Bioenerg. Biomembr., 2007, 39, 51-57.

52 Y. H. Wu and D. F. Swaab, Sleep Med., 2007, 8, 623-636.

53 G. C. Brainard, J. P. Hanifin, J. M. Greeson, B. Byrne, G. Glickman and E. Gerner, J. Neurosci., 2001, 21, 6405-6412. 54 J. Arendt, E. J. Van Someren, R. Appleton, D. J. Skene and T. Akerstedt, Clin. Med., 2008, 8, 381-383. 